\title{
Mental models as representations of text
}

\author{
ALAN GARNHAM \\ Centre for Research on Perception and Cognition, Laboratory of Experimental Psychology \\ University of Sussex, Brighton BNI 9QG, England
}

\begin{abstract}
A theory of text representation is outlined which suggests that a text is encoded in a mental model that contains representations of only those individuals and events that are relevant to the interpretation of the text in question. Such models are constructed on-line in response to cues in the text and with reference to knowledge about the world. An experiment is reported which demonstrates that mental models represent the events most probably described in a text. The results confirm previous findings that memory for the content of a passage is not based on the construction of one of its linguistically motivated representations. However, unlike most previous findings, they do support a positive proposal about the nature of the representations on which memory for content is based.
\end{abstract}

People usually process text with a view to extracting its content rather than its linguistic form. They try to create a representation of the information contained in it. One of the tasks for psycholinguistic research is to provide an explanatory theory of the nature of such representations and of the way in which they are constructed as texts are processed. In the 1960s, psycholinguists investigated the hypothesis that the mental encoding of a sentence corresponds to one of its linguistically motivated representations, its syntactic deep structure (see, e.g., Clifton, Kurcz, \& Jenkins, 1965; Gough, 1965; Mehler, 1963), or its semantic representation (see, e.g., Bregman \& Strasberg, 1968; Fillenbaum, 1966). This hypothesis was quickly proved incorrect. The experimental work of Bransford and his colleagues (e.g., Bransford, Barclay, \& Franks, 1972; Bransford \& Franks, 1971) showed that representations of sentences are influenced by knowledge of the world, which must be distinguished from semantic knowledge. Bransford claimed that representations of texts are built up by "constructive" processes, which "integrate" the material from several sentences. These ideas undoubtedly contain some truth, but they are not precise enough to be regarded as an explanatory theory of text representation.

Many psycholinguists have continued to use the term "semantic representation" for the encoding of the content of a passage (see, e.g., Foss \& Hakes, 1978). However, in most cases, use of this term does not imply a commitment to the view that memory for content should be explained by importing ideas from linguistics into psycholinguistic theories.

The work described in this paper is part of a doctoral thesis submitted to Sussex University. It was supported by a grant from the Science Research Council (Great Britain). I would like to thank Phil Johnson-Laird for many useful comments on earlier versions of the manuscript and Charles Clifton for a number of helpful suggestions.
The most clearly formulated view of semantics is that of Montague (1970). According to his account, which is currently gaining widespread favor among linguists, it is fairly clear that the mental encoding of a sentence cannot be a semantic representation. For Montague, the meaning of a sentence determines, for any situation, whether the sentence accurately describes that situation. Thus, a sentence's meaning determines the set of events that the sentence provides a true description of. However, the point of processing a sentence in context is not to determine which events it could be describing, but to focus on one particular member of that set and to represent it. The representation will contain items standing for particular individuals in the world, and it will be a representation of an event rather than of the meaning of a sentence. Sentences with different meanings may be used to describe the same event. Of course, the semantics of the sentence will be important in setting up the representation, just as syntax is needed to determine meaning. But neither syntax nor semantics need be retained in the final representation. Both may be, particularly by people who think that they are going to be asked about them, but usually neither will be.

If the representation of a text is not a representation of its semantics, how should it be characterized? The hypothesis that is examined in the present paper is that memory for the content of a passage is based on a mental model of the situation in a real or imaginary world that the passage describes (Johnson-Laird, 1980; Johnson-Laird \& Garnham, 1980). One aspect of such models is that they contain tokens that stand for individuals and these tokens are tagged with information about the properties of those individuals.

There is much empirical evidence to suggest that mental representations of texts contain such tokens (Anderson, 1977; Anderson \& Bower, 1973, chap. 9; Anderson \& Hastie, 1974; Ortony \& Anderson, 1977). These experiments show that coreferring singular terms 
are very often confused with each other. There are also a number of computer programs that create representations of texts that contain tokens standing for individuals (Anderson \& Bower, 1973; Rumelhart, Lindsay, \& Norman, 1972; Winograd, 1972), which lends further support to the idea that the representation of individuals is essential to comprehension.

However, to claim that texts are represented in mental models is not simply to say that representations contain tokens standing for individuals. Johnson-Laird and Garnham (1980) have shown that the interpretation of singular terms in a text depends crucially on an account of which individuals are possible referents of those terms. Thus, for each text, there is a corresponding mental model containing tokens standing for the individuals that the text is about. Johnson-Laird and Garnham also outline a theory of how cues in a text and knowledge of the world are used to decide when to introduce representations of individuals into a mental model. This work forms the basis of a theory of the way representations are constructed on-line, as successive parts of a text are read. (See Johnson-Laird \& Garnham for a more detailed account of the role of definite and indefinite descriptions in this process.)

Consider a text that contains the sentence "By the window was a man with a martini." The indefinite description "a man" introduces a new referent, and, given that no other men with martinis or standing by windows are introduced by the text, the sentence allows the reader to infer that, in the context of the passage, "the man with the martini" and "the man standing by the window" are coreferential. If either of these expressions occurs later in the passage, it can be assumed to refer to the individual previously introduced into the discourse model. Garnham (1981) showed that if, later in the passage, a sentence such as "The man standing by the window shouted to the host" occurred, subjects were unable to remember whether they had heard that sentence or "The man with the martini shouted to the host." Thus people confuse semantically different expressions that are established as being coreferential in the context of a particular passage.

A mental model contains not only representations of a particular set of individuals, but also representations of a particular series of events. Just as an individual may be described in various ways, so may an event be related in different sentences. Those sentences may have different semantics. That is to say, the sets of events that they could be used to describe may be very different. Nevertheless, in context, they may clearly describe the same event, if that event lies in the intersection of those sets.

If the idea of mental models is correct, then it should be possible to show that text representations are organized around representations of events, rather than linguistic expressions describing those events. The experiment to be described in this paper addresses the question of whether people confuse prepositional phrases that have very different semantic functions, if the sentences in which those phrases occur probably describe the same situation in the world. Consider the sentences "The hostess bought a mink coat from the furrier" and "The hostess bought a mink coat in the furrier's." They would usually be used to describe the same event, but they need not be. It is unusual to buy a mink coat in the furrier's but not from the furrier, but it is possible. One could be bought from another customer, for example. Similarly, it is possible to buy a coat from the furrier, but not at his place of business. The sets of events that the two sentences could be used to describe have, perhaps, a relatively small intersection, but that intersection contains most, if not all, of the most probable of those events. If knowledge about the relative probabilities of events affects the kinds of representations that are set up when sentences are processed, then the two sentences above should receive fairly similar representations. However, if representations are affected only by the sets of events that the sentences might possibly describe (i.e., the semantics of the sentences), then the representations should be markedly different.

Another pair of sentences that differ from each other in surface form in the same way as the pair above is "The hostess received a telegram from the furrier" and "The hostess received a telegram in the furrier's." These two sentences would usually describe different events, but they could describe the same event. It is possible to be in the furrier's and to get a telegram from him, but it is unlikely. The intersection of the two sets of events that the sentences could describe is again fairly small, but in this case the events in the intersection are improbable ones. If the representations of the sentences are merely determined by the sets of events they could describe, then the difference between the members of the second pair should be of the same order as that between the members of the first pair, and there would be no reason to expect more confusions in memory in one case than in the other. However, if knowledge of probabilities affects the representations, then the second two sentences should have relatively distinct encodings that should not be confused in memory, whereas confusions between the first two sentences would be expected.

\section{METHOD}

\section{Subjects}

The subjects were 12 volunteers from the student population of Sussex University. They were paid 60 pence for participating in the experiment, which lasted about $25 \mathrm{~min}$.

\section{Materials}

Twenty-four sets of four sentences were constructed. An example of one of the sets is "The girl was given a complete pedicure at the chiropodist's. The girl was given a complete pedicure by the chiropodist. The girl had her handbag stolen 
at the chiropodist's. The girl had her handbag stolen by the chiropodist." In each set of sentences, there were two pairs, each of which differed only in the final prepositional phrase. In 12 of the sets, the contrasted phrases were "at" and "by" phrases, for example, "at the chiropodist's" and "by the chiropodist." In the other 12 sets, they were "in" and "from" phrases, such as "in the furrier's" and "from the furrier."

The "at" and "in" phrases were all what would be called, in traditional school grammar, adverbial phrases of place. In Fillmore's (1968) case grammar, they are called "locatives," since they indicate the location at which an event occurs. The "by" phrases were all of the kind that indicates an underlying subject in a passive sentence. The "from" phrases also indicate participants in actions. Fillmore's original system of cases is not well suited to dealing with sentences that describe situations with two active participants, although a revised version (Fillmore, 1971) fares a lot better. Quirk, Greenbaum, Leech, and Svartvik (1972) classify the use of "from the furrier" in the above sentence as indicating a purposive source or origin of an action. Such a phrase is semantically very different from "in the furrier's."

The sentences in each pair were, therefore, semantically distinct, because of their respective final prepositional phrases. The semantic difference was approximately the same for each pair in a set, as their wording differed in the same way. However, for a pair of sentences such as "The girl was given a complete pedicure at the chiropodist's" and "The girl was given a complete pedicure by the chiropodist," the semantic contrast does not give rise to a difference in the probable significance (Johnson-Laird, 1977, pp.105-109) of the two sentences. A pedicure performed by a chiropodist is very likely to be done at the chiropodist's and vice versa. Such a pair of sentences will be referred to as a confusable pair. The same semantic contrast between "The girl had her handbag stolen at the chiropodist's" and "The girl had her handbag stolen by the chriopodist" does lead to a difference in probable significance, because a chiropodist is unlikely to steal his customer's property. This kind of sentence pair will be called a nonconfusable pair.

In order that the four sentences in each set of materials could be presented to different subjects, four lists were constructed. The lists were recorded on a B\&O tape recorder with the author as speaker. The full set of materials is given in the Appendix.

The memory test contained 24 four-alternative forced-choice (4AFC) items in a five-page booklet. There was one item related to each set of sentences; it comprised the four sentences in that set in random order.

In a further test, subjects were asked to make judgments about whether pairs of sentences from the sentence sets would usually be used to describe the same situation. Each test item comprised two sentences from one of the sets, differing only in the final prepositional phrase. Under each pair of sentences were the numbers $1-7$, to be used as a scale on which subjects were to rate similarity. The tests were presented in three-page booklets.

\section{Design}

The four presentation lists were designed so that each subject would hear equal numbers of sentences from confusable and nonconfusable pairs and so that they would hear equal numbers of sentences of each type from "at/by" and "from/in" material sets. In addition to the 24 test sentences, each list contained 12 filler items, which were the same for all the lists. The 12 filler items comprised 6 sentences ending with a prepositional phrase introduced by "under," for example, "under the bed," and 6 sentences ending with a prepositional phrase such as "over the fields." The order of the 36 items was determined separately for each list and was subject to the following constraints: (1) The first 2 items were chosen at random from the 12 filler items. (2) The last 2 items were chosen at random from the remaining
10 filler items. (3) Items 3-34 were the remaining 8 filler items and the 24 test items in random order.

The memory test was the same for all subjects. The order of the four sentences in each test item was randomized independently, and the order of the 24 'items was also randomized.

There were two different versions of the test in which subjects had to make judgments about the sentences. Each test contained 24 pairs of sentences, 1 from each set of materials. Twelve of the items in each test were confusable pairs and 12 were nonconfusable pairs. There were equal numbers of each kind of pair with "at/by" phrases and with "from/in" phrases. The order of the two sentences in each test item was randomized separately. The order of the 24 items was randomized separately for each of the tests, but it was the same for all subjects who were given that test.

Each subject heard one and only one presentation list and, therefore, heard one and only one sentence from each set of materials. Equal numbers of subjects heard each of the four lists. All subjects heard sentences from confusable and nonconfusable pairs, so this factor was both within subjects and within material sets.

In the rating test at the end of the experiment, each subject made judgments about both kinds of sentence pair, so that a test could be made of whether they made more confusions between sentences that they judged to be more confusable. The factor was again within subjects and within material sets.

\section{Procedure}

Subjects were tested individually. They heard one of the presentation lists in a small room. As they listened to the sentences, they were asked to rate how easily they could imagine the situation described by each sentence. A 7-point scale was used, with 1 meaning easy to imagine. No mention was made of the subsequent tests. These instructions were used to encourage subjects to consider the sentences as descriptions of events, rather than simply as items to memorize.

Presentation of the list was immediately followed by an interpolated task designed to produce some forgetting and to prevent the use of very short-term memory. Subjects were given a sheet of 48 three-digit number plus three-digit number (e.g., $654+497=$ ?) addition problems. They were told to work through them fairly rapidly and were given up to $10 \mathrm{~min}$ to complete them. If they finished before that time, they moved straight on to the recognition test. (There is evidence to suggest that decay of short-term memory traces depends not on elapsed time, but on what other tasks have been performed; see, e.g., Baddeley \& Hitch, 1977.)

The recognition test followed the interpolated task immediately. Subjects were asked to work fairly rapidly through the 24 4AFC tests and not to go back and alter previous responses. There was no time limit.

The second test followed the recognition test immediately. Subjects were asked "to consider the kinds of situation which each sentence in the pair can be used to describe, and to make a judgment about how similar these kinds of situation are."

\section{RESULTS}

\section{Similarity Judgments}

The results of the similarity ratings will be presented first, since these validate the materials. Each subject made 12 judgments about confusable pairs of sentences and 12 about nonconfusable pairs. All subjects judged the situations described by the confusable pairs to be more similar. The overall mean scores were 2.02 for confusable pairs and 5.06 for nonconfusable pairs (7-point scale). Mann-Whitney U tests were performed on the ratings for each subject. In every case, the differ- 
ence between the two kinds of pair was significant ( $p<.025$ for one subject, $p<.01$ for one subject, $\mathrm{p}<.005$ for one subject, and $\mathrm{p}<.001$ for nine subjects].

It is clear from these results that the subjects in the experiment were in general agreement with the author about whether pairs of sentences would usually be used to describe the same situation. The instructions were carefully worded in an attempt to ensure that subjects did not base their judgments on some preconceived notion of sentence meaning, but on the range of situations to which the sentence could be applied and the relative probabilities that it would be so applied.

\section{Recognition Tests}

Responses in the recognition test were classified as correct, incorrect, or confusions. A response was correct if the sentence chosen in the test was the one that had been presented. A confusion was deemed to have occurred if the sentence chosen was from the same pair as the presented sentence, that is to say, if it differed from the presented sentence only in its final prepositional phrase. A response was incorrect if a subject chose a sentence from the other pair in the set, for example, if he or she chose either "The worker had her prescription made up by the chemist" or "The worker had her prescription made up at the chemist's" when the presented sentence was "The worker was made to look foolish by the chemist" or "The worker was made to look foolish at the chemist's."

Table 1 shows the number of responses of each kind for presented sentences from confusable and nonconfusable pairs. As can be seen from the table, the majority of responses, about $65 \%$, were correct and fewer than $5 \%$ were incorrect. The average number of confusions per subject, of a possible 12, was 4.3 for confusable pairs and 2.8 for nonconfusable pairs. Eight of the 12 subjects made more confusions for the confusable pairs (three ties; Wilcoxon's $\mathrm{T}=7.5, \mathrm{n}=9, \mathrm{p}<.05$, onetailed). For 16 of the 24 sets of materials, more confusions were made between the confusable pair (four ties; Wilcoxon's $\mathrm{T}=51.5, \mathrm{n}=20, \mathrm{p}<.025$, one-tailed).

Table 1

Total Number of Responses of Each Kind in the Recognition Test as a Function of the Kind of Pair From Which the Presented Sentence Was Drawn

\begin{tabular}{lccc} 
& \multicolumn{3}{c}{$\begin{array}{c}\text { Kind of Pair From Which Presented } \\
\text { Sentence Was Drawn }\end{array}$} \\
\cline { 2 - 4 } Response & Confusable & Nonconfusable & Total \\
\hline Correct & 87 & 102 & 189 \\
Confusion & 52 & 34 & 86 \\
Incorrect & 5 & 8 & 13 \\
Total & 144 & 144 & 288 \\
\hline
\end{tabular}

Note-Each entry represents 12 subjects and has a maximum value of 144. Column totals must be 144.

\section{Rated Confusability and Actual Confusions}

Stronger support for the idea that texts are encoded in mental models of situations would be obtained if the rated similarity of the situations described by pairs of sentences predicts the number of confusions between those sentences in the recognition test. In order to check for such a correlation, Spearman's rho was calculated between the confusability scores and the number of experimentally induced confusions for each set of materials (pooled in each case over 12 subjects). Separate tests were made for the confusable and nonconfusable pairs. For the confusable pairs, there was a significant correlation (rho $=-.44, \mathrm{p}<.025$, one-tailed). The value is negative because subjects were asked to give numerically small scores to pairs of sentences that would be used to describe the same situation. For nonconfusable pairs, there was no significant correlation $($ rho $=.02)$.

\section{DISCUSSION}

The results of the experiment show that the same semantic difference between two sentences does not always give rise to the same amount of confusion about which of the two sentences has previously been encountered. For pairs of sentences designated confusable before the experiment, because they probably described the same situation, the degree of experimentally induced confusion was related to the judged similarity of the range of situations that the sentences would be likely to describe. Such a correlation was not found for sentences judged likely to describe different situations. The most probable explanation for this lack of correlation is that the experimentally produced confusion between nonconfusable pairs arose only when subjects failed to encode the sentences properly and had to rely in part on guessing in the recognition tests. Proper encodings of sentences from nonconfusable pairs are probably distinct enough not to be confused in memory. The findings cannot be accounted for semantically, because the semantic difference between "The leader purchased three loaves from the baker" and "The leader purchased three loaves at the baker's" is comparable to that between "The leader got a punch on the nose from the baker" and "The leader got a punch on the nose at the baker's." Sentences within each pair can be used to describe wholly disparate situations or closely similar ones. What differentiates the two pairs is the relative probabilities with which they will be used to do so. Knowledge about the probabilities of events referred to in a sentence cannot be included in the semantics of that sentence, yet it does appear to affect the representations of sentences that people construct when they hear those sentences. The precise way in which probabilities affect representation is not made clear by the experiment, but the results 
are sufficient to rule out a semantically based account of the encoding of sentences.

It has been suggested, by a reviewer, that an alternative explanation of the results is that the prepositional phrases in the confusable pairs tend to be forgotten, because they are redundant. Loaves are typically purchased at a baker's, so it is unnecessary to encode the final prepositional phrase. However, this explanation is not really an alternative, for in order to work out that "from the baker" and "at the baker's" are redundant, it is necessary to compute the events most probably described by the sentences in the confusable pairs. Thus, at some stage in the comprehension process, subjects construct a nonsemantic representation of those events.

\section{GENERAL DISCUSSION}

Psycholinguists realized some time ago that memory for the content of a passage should not be equated with memory for its semantic representation as described by linguists. Nevertheless, detailed theories about the representations on which memory for content is based were not developed. In this paper, such a theory has been outlined, the theory that texts are represented in mental models. These models are structurally similar to parts of real or imaginary worlds that are described in the texts from which they derive. They are not necessarily structurally similar to any linguistic representation of the text-syntactic, lexical, or semantic.

It is assumed that a formal account can be given of how such models are built up piecewise as text is processed. Johnson-Laird and Garnham (1980, pp.379. 382) outline part of such an account, considering the case of definite and indefinite descriptions. These expressions are regarded as cues for the introduction of new tokens into mental models. It is further assumed that there is some way of deciding whether a mental model constructed in response to a text is a correct representation of the world. In other words, given the model that has been constructed from a text, there is a way of deciding whether the text that it represents is true (see Kamp, Note 1). A mental model is a representation of the world and corresponds roughly to what Kamp calls a "discourse representation." Thus, it is not, strictly speaking, a model in the sense of model-theoretic semantics. It is not a set-theoretic construct, but rather, something that represents such a construct. However, a mental model stands in a very simple relation to the things in the world that comprise a model in formal semantics, in much the same way that a symbolic representation of a model in a treatise on semantics does, and, as Johnson-Laird and Garnham (1980) point out, it is often convenient to think of language as being about a mental model rather than about the world. A mental model is a surrogate for the world in a way in which a piece of prose can never be.
Although most versions of formal semantics (e.g., Montague, 1973) have appeared logically and psychologically inadequate for dealing with texts rather than single sentences, Kamp's (Note 1) work shows how the apparatus of model theory can be applied to discourse and suggests how mental models can be related to the models of formal semantics. It must be stressed, however, that the discovery of such a relation is very different from equating mental models with semantic representations.

\section{REFERENCE NOTE}

1. Kamp, J. A. W. A theory of truth and semantic representation (technical report). Austin: University of Texas, Center for Cognitive Science, August 1980.

\section{REFERENCES}

Anderson, J. R. Memory for information about individuals. Memory \& Cognition, 1977, 5, 430-442.

Anderson, J. R., \& Bower, G. H. Human associative memory. Washington, D.C: Winston, 1973.

Anderson, J. R., \& Hastie, R. Individuation and reference in memory: Proper names and definite descriptions. Cognitive Psychology, 1974, 6, 495-514.

Baddeley, A. D., \& Hitch, G. J. Recency re-examined. In S. Dornic (Ed.), Attention and performance VI. Hillsdale, N.J: Erlbaum, 1977.

Bransfond, J. D., Barclay, J. R., \& Fannks, J. J. Sentence memory: A constructive versus interpretive approach. Cognitive Psychology, 1972, 3, 193-209.

Bransford, J. D., \& Franks, J. J. The abstraction of linguistic ideas. Cognitive Psychology, 1971, 2, 331-350.

Bregman, A., \& Strasberg, R. Memory for the syntactic form of sentences. Journal of Verbal Learning and Verbal Behavior, $1968,7,396-403$.

Chifton, C., Kurcz, I., \& Jenkins, J. J. Grammatical relations as determinants of sentence similarity. Journal of Verbal Learning and Verbal Behavior, 1965, 4, 112-117.

Fildenbaum, S. Memory for gist: Some relevant variables. Language and Speech, 1966, 9, 217-227.

Fillmore, C. J. The case for case. In E. Bach \& R. T. Harms (Eds.), Universals in linguistic theory. New York: Holt, Rinehart, \& Winston, 1968.

Fillmone, C. J. Types of lexical information. In D. D. Steinberg \& L. A. Jakobovits (Eds.), Semantics: An interdisciplinary reader in philosophy, linguistics and psychology. Cambridge: Cambridge University Press, 1971.

Foss, D. J., \& Hakes, D. T. Psycholinguistics: An introduction to the psychology of language. Englewood Cliffs, N.J: PrenticeHall, 1978.

GARNHAM, A. Mental models as representations of discourse and text. Unpublished doctoral thesis, Sussex University, 1981.

GougH, P. B. Grammatical transformations and speed of understanding. Journal of Verbal Learning and Verbal Behavior, $1965,4,107-111$.

Johnson-Laird, P. N. Psycholinguistics without linguistics. In N. S. Sutherland (Ed.), Tutorial essays in psychology (Vol. 1). Hillsdale, N.J: Erlbaum, 1977.

Johnson-LaIRd, P. N. Mental models in cognitive science. Cognitive Science, 1980, 4, 71-115.

Johnson-Laind, P. N., \& Gahnham, A. Descriptions and discourse models. Linguistics and Philosophy, 1980, 3, 371-393.

MEHLER, J. Some effects of grammatical transformations on the recall of English sentences. Journal of Verbal Learning and Verbal Behavior, 1963, 2, 346-351. 
Montague, R. Universal grammar. Theoria, 1970, 36, 373-398. Montaque, $R$. The proper theory of quantification in ordinary English. In J. Hintikka, J. Moravcsik, \& P. Suppes (Eds.), Approaches to natural language. Dordrecht: Reidel, 1973.

Ortony, A., \& Anderson, R. C. Definite descriptions and semantic memory. Cognitive Science, 1977, 1, 174-183.

Quirk, R., Greenbaum, S., Leech, G. N., \& Svartvik, J. A grammar of contemporary English. London: Longman, 1972.

Rumelhart, D. E., Lindsay, P. H., \& Norman, D. A. A process model for long-term memory. In E. Tulving \& W. Donaldson (Eds.), Organization of memory. New York: Academic Press, 1972.

Winograd, T. Understanding natural language. Cognitive Psychology, 1972, 3, 1-191.

\section{APPENDIX \\ MATERIALS USED IN THE EXPERIMENT}

In each set of sentences, $a$ and $b$ are the confusable pair and $c$ and $d$ are the nonconfusable pair.

1. (a) The girl was given a complete pedicure at the chiropodist's. (b) The girl was given a complete pedicure by the chiropodist. (c) The girl had her handbag stolen at the chiropodist's. (d) The girl had her handbag stolen by the chiropodist.

2. (a) The clerk was going to have the wood cut up for him at the joiner's. (b) The clerk was going to have the wood cut up for him by the joiner. (c) The clerk was informed about the rail strike at the joiner's. (d) The clerk was informed about the rail strike by the joiner.

3. (a) The judge got his contact lenses from the optician. (b) The judge got his contact lenses in the optician's. (c) The judge answered a telephone call from the optician. (d) The judge answered a telephone call in the optician's.

4. (a) The leader purchased three loaves from the baker. (b) The leader purchased three loaves in the baker's. (c) The leader got a punch on the nose from the baker. (d) The leader got a punch on the nose in the baker's.

5. (a) The salesman was given two pounds for the coat at the pawnbroker's. (b) The salesman was given two pounds for the coat by the pawnbroker. (c) The salesman was bumped into on the step at the pawnbroker's. (d) The salesman was bumped into on the step by the pawnbroker.

6. (a) The teacher wanted her clock repaired at the watchmaker's. (b) The teacher wanted her clock repaired by the watchmaker. (c) The teacher was saved from falling down at the watchmaker's. (d) The teacher was saved from falling down by the watchmaker.

7. (a) The politician bought his wife a ring from the jeweller. (b) The politician bought his wife a ring in the jeweller's. (c) The politician received congratulations from the jeweller. (d) The politician received congratulations in the jeweller's.

8. (a) The bishop bought a new pipe from the tobacconist. (b) The bishop bought a new pipe in the tobacconist's. (c) The bishop heard an account of the state of the nation from the tobacconist. (d) The bishop heard an account of the state of the nation in the tobacconist's.

9. (a) The servant had a tooth removed at the dentist's. (b) The servant had a tooth removed by the dentist. (c) The servant was injured on the knee at the dentist's. (d) The servant was injured on the knee by the dentist.

10. (a) The student bought some spring cabbage from the greengrocer. (b) The student bought some spring cabbage in the greengrocer's. (c) The student heard about the concert from the greengrocer. (d) The student heard about the concert in the greengrocer's.
11. (a) The writer collected his manuscript from the publisher. (b) The writer collected his manuscript in the publisher's. (c) The writer got an invitation to a wedding from the publisher. (d) The writer got an invitation to a wedding in the publisher's.

12. (a) The matron got three pork chops from the butcher. (b) The matron got three pork chops in the butcher's. (c) The matron heard an account of the cricket match from the butcher. (d) The matron heard an account of the cricket match in the butcher's.

13. (a) The worker had her prescription made up at the chemist's. (b) The worker had her prescription made up by the chemist. (c) The worker was made to look foolish at the chemist's. (d) The worker was made to look foolish by the chemist.

14. (a) The chairman bought a cup for the prize from the silversmith. (b) The chairman bought a cup for the prize in the silversmith's. (c) The chairman heard about the epidemic from the silversmith. (d) The chairman heard about the epidemic in the silversmith's.

15. (a) The vicar went to have a suit fitted at the tailor's. (b) The vicar went to have a suit fitted by the tailor. (c) The vicar was given an old lampshade at the tailor's. (d) The vicar was given an old lampshade by the tailor.

16. (a) The actress was given a shampoo and set at the hairdresser's. (b) The actress was given a shampoo and set by the hairdresser. (c) The actress's brother was discovered at the hairdresser's. (d) The actress's brother was discovered by the hairdresser.

17. (a) The woman was given a thorough examination at the doctor's. (b) The woman was given a thorough examination by the doctor. (c) The woman was told about the new traffic scheme at the doctor's. (d) The woman was told about the new traffic scheme by the doctor.

18. (a) The secretary bought some curtains from the draper. (b) The secretary bought some curtains in the draper's. (c) The secretary got an invitation to a party from the draper. (d) The secretary got an invitation to a party in the draper's.

19. (a) The husband bought some sugar from the grocer. (b) The husband bought some sugar in the grocer's. (c) The husband received a message from the grocer. (d) The husband received a message in the grocer's.

20. (a) The policeman had a bouquet made up at the florist's. (b) The policeman had a bouquet made up by the florist. (c) The policeman was attacked from behind at the florist's. (d) The policeman was attacked from behind by the florist.

21. (a) The hostess bought a mink coat from the furrier. (b) The hostess bought a mink coat in the furrier's. (c) The hostess received a telegram from the furrier. (d) The hostess received a telegram in the furrier's

22. (a) The farmer bought his copy of "Farmer's Weekly" from the newsagent. (b) The farmer bought his copy of "Farmer's Weekly" in the newsagent's. (c) The farmer borrowed a pound from the newsagent. (d) The farmer borrowed a pound in the newsagent's.

23. (a) The academic took a book to be bound at the bookbinder's. (b) The academic took a book to be bound by the bookbinder. (c) The academic was taken to an exhibition at the bookbinder's. (d) The academic was taken to an exhibition by the bookbinder.

24. (a) The detective was advised not to go and inspect the house at the estate-agent's. (b) The detective was advised not to go and inspect the house by the estate-agent. (c) The detective was tipped off at the estate-agent's. (d) The detective was tipped off by the estate-agent.

(Received for publication December 29, 1980; revision accepted May 29, 1981.) 